\title{
Epidemiology, clinical characteristics and outcome of acute kidney injury in intensive care units of alexandria university hospitals
}

\begin{abstract}
Background: Acute kidney injury (AKI) considered as a major public health problem that affects millions of patients worldwide and leads to decreased survival and increased progression of chronic kidney disease (CKD).
\end{abstract}

Aim of study: Epidemiology, clinical characteristics and outcome of acute kidney injury in patients admitted to main Alexandria university hospitals over six months.

Methods: All patients who were admittedto intensive care units (ICUs) at Alexandria University Hospitals wereprospectively studied. Patients whodeveloped ICU-acquired acute renalfailure were collected in the period over six months.

Results: Our study included 500 patients in general ICU. General ICU patients classified according to renal impairment: 303 cases (no AKI) $60.6 \%$, 74 cases (CKD) $14.8 \%, 55$ cases (AKI) 11\%, 38 cases (acute on top of CKD) 7.6\%, 28 cases (ESRD) 5.6\%, 2 cases (obstructive uropathy) $0.4 \%$. Bimodal distribution of age in patients developed AKI at (18-30y) \& (62-85y). 75 Toxicological cases, 3 developed AKI ( 2 organo phosphorus \& 1 scorpion bite). The most common cause of AKI in our study was septic AKI $60 \%$ \& among 128 cases of sepsis $32 \%$ not developed AKI $25.8 \%$ developed septic AKI $32 \%$ acute on top of CKD/10.2\% ESRD. Mortality rate allover general ICU patients was 140/500 (28\%) while allover AKI patients in general ICU 30/55(54\%) and allover septic AKI 26/33(79\%).

Conclusion: AKI is a worsening problem, but its true incidence is in need of huge work, our work was $1^{\text {st }}$ up to our knowledge in Alexandria to check its incidence hence planning for better outcome.

Keywords: acute kidney injury, ICU, Alexandria, Egypt
Volume 3 Issue 6 - 2016

\section{Rasha Shafie, Iman Elgohary, Sherif Hegab, Nahed Baddour, Neveen Lewis, Ahmed Adam Internal Medicine Department, Alexandria University, Egypt}

\section{Correspondence: Rasha Shafie, Internal Medicine Department, Nephrology and kidney transplantation Unit Faculty of Medicine, Alexandria University, Egypt, Email rasha_shafie@yahoo.com}

Received: November II, 2016 | Published: December 30 2016
Abbreviations: AKI, Acute kidney injury; CKD, chronic kidney disease; ICUs, intensive care units; ADQI, acute quality dialysis initiative; RRT, renal replacement therapy; AKIN, acute kidney injury network; TCA, tricyclic antidepressant

\section{Introduction}

Acute kidney injury (AKI) is a common clinical problem encountered in critically ill patients and characteristically portends an increase in morbidity and mortality. ${ }^{1}$ Previous epidemiologic investigations describing the incidence and outcomes of AKI in critically ill patients have been limited due to the differences used in defining and classifying AKI. ${ }^{2}$ This has been unfortunate and likely contributed to hindering scientific progress in the field of critical care nephrology. ${ }^{3}$ The RIFIE criteria, a consensus definition for AKI, were published by the acute Quality Dialysis initiative (ADQI) work group in $2004 .{ }^{4}$ These criteria have been validated in several clinical settings and shown to correlate with important outcomes such as need for renal replacement therapy (RRT), length of hospital stay, and mortality. In fact, the RIFIE criteria are now well recognized and have been cited in more than 150 manuscripts.
More recently, the Acute Kidney Injury Network (AKIN) group, an international collaboration of nephrologists and intensivists, has proposed refinements to the RIFLE criteria. ${ }^{6}$ In particular, the AKIN group sought to increase the sensitivity of the RIFLE criteria by recommending that a smaller change in serum creatinine $(\geq 26.2$ $\mu \mathrm{mol} / \mathrm{L}$ ) can be used as a threshold to define the presence of AKI and identify patients with Stage 1 AKI (analogous to RIFLE-Risk). Second, a time constraint of $48 \mathrm{~h}$ for the diagnosis of AKI was proposed. Finally, any patients receiving renal replacement therapy (RRT) were now to be classified as Stage 3 AKI (RIFLE-Failure). It is currently unknown whether discernible advantages exist with one approach to definition and classification versus the other. ${ }^{7}$

\section{Methods}

For each patient with ICU acquired acute kidney injury, Details and variables such as age, sex, cause of admission, RRT, and outcome were collected. AKI was defined and classified by means of AKIN and RIFLE criteria (Table $1 \&$ Table 2).

Table I Risk, Injury, Failure, Loss of function and End stage kidney disease (RIFLE) staging system

\section{RIFLE Serum creatinine criteria}

Risk $\quad \mathrm{SCr} \geq 1.5 \mathrm{X}$ baseline

Urine output criteria

$<0.5 \mathrm{~mL} / \mathrm{kg} / \mathrm{h}>6 \mathrm{~h}$ 
Table continued

\begin{tabular}{llc}
\hline RIFLE & Serum creatinine criteria & Urine output criteria \\
\hline Injury & $\mathrm{SCr} \geq 2.0 X$ baseline & $<0.5 \mathrm{~mL} / \mathrm{kg} / \mathrm{h}>12 \mathrm{~h}$ \\
Failure & $\mathrm{SCr} \geq 3.0 \times$ baseline or serum creatinine $\geq 4 \mathrm{mg} / \mathrm{dL}$ with an absolute increase of $>0.5 \mathrm{mg} / \mathrm{dL}$ & $<0.3 \mathrm{~mL} / \mathrm{kg} / \mathrm{h}>24 \mathrm{~h} \mathrm{or} \mathrm{anuria}>12 \mathrm{~h}$ \\
Loss & Complete loss of kidney function $>4 \mathrm{wks}$ & \\
ESK & End-stage kidney disease $>3$ months & \\
\hline
\end{tabular}

Table 2 The Acute Kidney Injury Network (AKIN) staging system

\begin{tabular}{|c|c|c|}
\hline AKIN & Serum creatinine criteria & $\begin{array}{l}\text { Urine output } \\
\text { criteria }\end{array}$ \\
\hline Stage I & $\mathrm{SCr} \geq \mathrm{I} .5 \mathrm{X}$ baseline or increase in $\mathrm{SCr} \geq 0.3 \mathrm{mg} / \mathrm{dl}$ from baseline & $<0.5 \mathrm{~mL} / \mathrm{kg} / \mathrm{h}>6 \mathrm{~h}$ \\
\hline Stage 2 & $\mathrm{SCr} \geq 2.0 \times$ baseline & $<0.5 \mathrm{~mL} / \mathrm{kg} / \mathrm{h}>12 \mathrm{~h}$ \\
\hline Stage 3 & $\begin{array}{l}\mathrm{SCr} \geq 3.0 \times \text { baseline or serum creatinine } \geq 4.0 \mathrm{mg} / \mathrm{dl}(\geq 354 \mu \mathrm{mol} / \mathrm{l}) \text { with an acute increase of at least } 0.5 \\
\mathrm{mg} / \mathrm{dl}(44 \mu \mathrm{mol} / \mathrm{l}) \text { or initiated on RRT (irrespective of stage at time of initiation) }\end{array}$ & $\begin{array}{l}<0.3 \mathrm{~mL} / \mathrm{kg} / \mathrm{h}>24 \mathrm{~h} \text { or } \\
\text { anuria }>12 \mathrm{~h}\end{array}$ \\
\hline
\end{tabular}

\section{Results}

\section{Base line characteristics}

Our study included 500 General ICU patients (classified according to renal impairment) (Figure 1) 303 no AKI (60.6\%), 74 CKD (14.8\%), 55 AKI (11\%), 38 acute on top of CKD (7.6\%), 28 ESRD (5.6\%), 2 obstructive uropathy $(0.4 \%)$ and Classified according to cause of admission (Figure 2) RTA 36 cases, Fall from height 30 cases, Toxo 60 cases, Botulism 10 cases, Snake bite 4 cases, Scorpion bite 1 case, Ischemic stroke 31 cases, Hemorrhagic stroke 25 cases, Meningitis 5 cases, Sepsis 128 cases, Hepatic 30 cases, DKA 26 cases, Obstetric 7 cases, Malignancy 21 cases, COPD 18 cases, IBD 1 case, GB 6 cases, TTP 2 cases, Cardiogenic 37 cases, Interstitial lung diseases 3 cases, Polymyositis 2 cases, Duchine muscle atrophy 1 case, Perforated peptic ulcer 1 case, Lupus pneumonitis 7 cases ,Myasthenia gravis 2 cases, Auto immune hemolytic anemia 1 case, Blood diathesis 2 cases, CP 2 cases, Mucopolysaccharidosis 1 case.

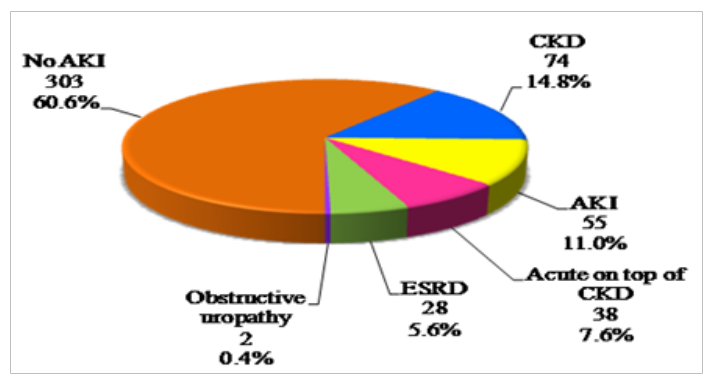

Figure I General ICU patients (classification according to renal impairment): (500 cases).

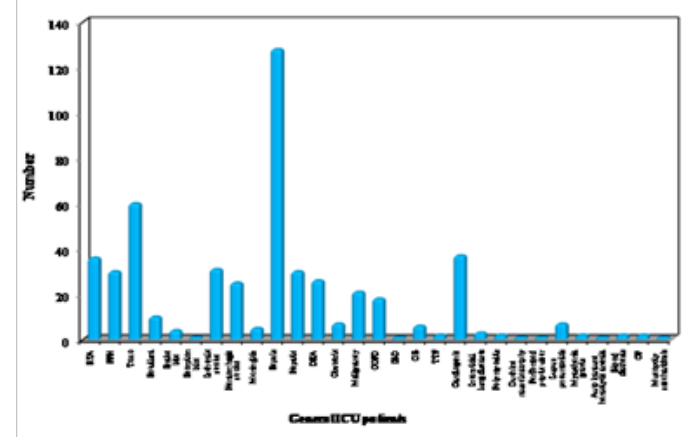

Figure 2 General ICU patient's classification regard cause of admission (500 cases).
Toxicological cases (75 cases) classified in to: (Figure 3) Botulism 10 cases, Scorpion bite 1 case (developed AKI), Snake bite 4 cases, Organo phosphorus 27 cases (2 developed AKI), Tricyclic antidepressant (TCA) 4 cases, Tegretol 2 cases, Formalin 1 case, Depakin 1 case, Kerosin 2 cases, Zn phosphate 3 cases, Calmipam 4 cases, Insulin for suiscidal purpose 2 cases, Lanoxin 6 cases, Tamol 1 case, Theophylline 1 case, Methanol 6 cases Distribution of AKI in general ICU according to sex: (Figure 4) Male patients 29/55 (52.7\%) Female patients 26/55 (47.3\%). Distribution of AKI in general ICU according to age (Figure 5) (Table 3).

Table 3 Distribution of general ICU AKI patients according to age

\begin{tabular}{ll} 
Age & No of patients \\
\hline $18-30 y$ & 13 \\
$31-39 y$ & 7 \\
$43-50 y$ & 7 \\
$52-60 y$ & 11 \\
$62-85 y$ & 17 \\
total & 55 cases
\end{tabular}

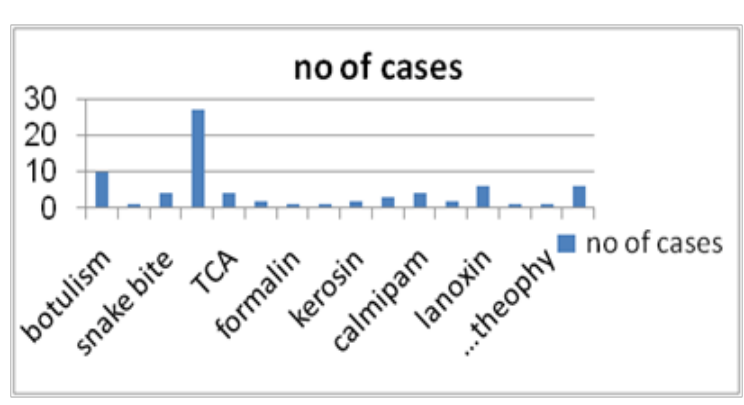

Figure 3 Toxicological ICU patients. 


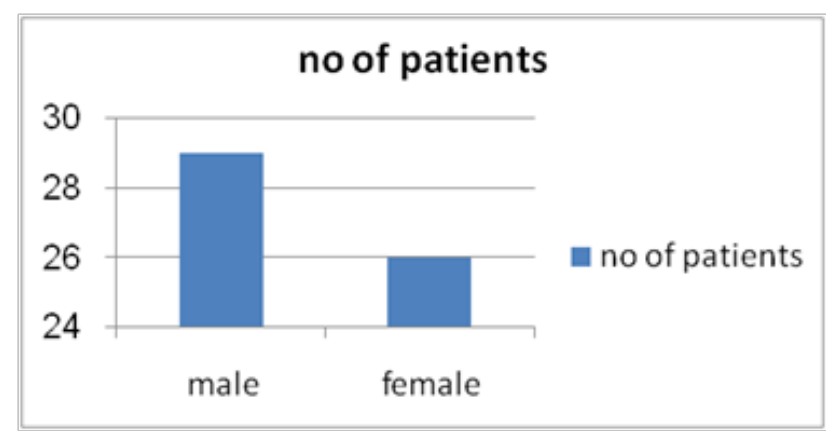

Figure 4 Distribution of general ICU AKI patients according to sex.

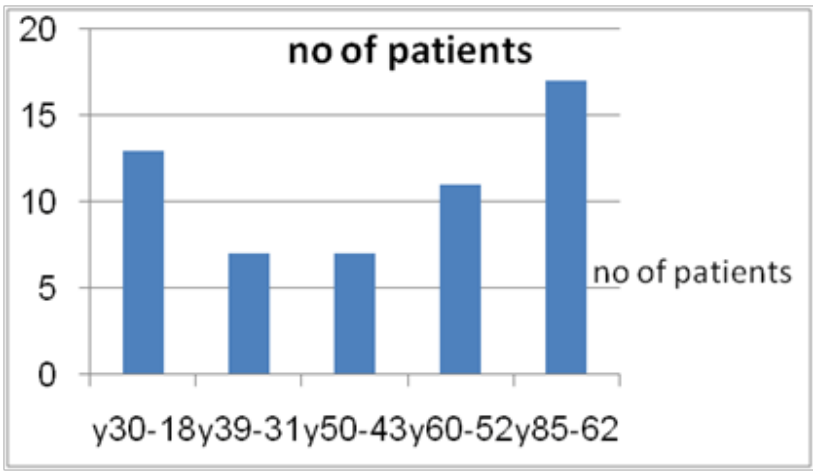

Figure 5 Distribution of general ICU AKI patients according to age.

General ICU AKI patients classified in to (Figure 6): septic AKI (33/55) 60\%, ischemic ATN (17/55) 31\%, cholemic nephrosis $(1 / 55) 1.8 \%$, rhabdomyolysis $(1 / 55) 1.8 \%$, cardio renal syndrome (1/55) $1.8 \%$, scorpion bite (1/55) $1.8 \%$, gastroenteritis (1/55) $1.8 \%$. According to RIFLE criteria acute kidney injury occurred in 55 patients $(11 \%)$, with category Risk in 20 patients $(36.4 \%)$, Injury in 27 patients $(49.1 \%)$ and failure in 8 patients $(14.5 \%)$. According to AKIN criteria acute kidney injury occurred in 55 patients (11\%), with stage 1 in 17 patients $(31 \%)$, stage 2 in 30 patients $(54.5 \%)$ and stage 3 in 8 patients $(14.5 \%)$. Mortality rates were $41.2 \%, 60 \%$, and $62.5 \%$ in AKIN stage 1, stage 2, and stage 3 groups, respectively. There is progressive increase in mortality rate across different classes of AKIN staging system. Mortality rate in class R was $40 \%$, in class I was $63 \%$, while in class $\mathrm{F}$ was $62.5 \%$, there is progressive increase in mortality rate across different classes of RIFLE staging system (Tables 4-6).

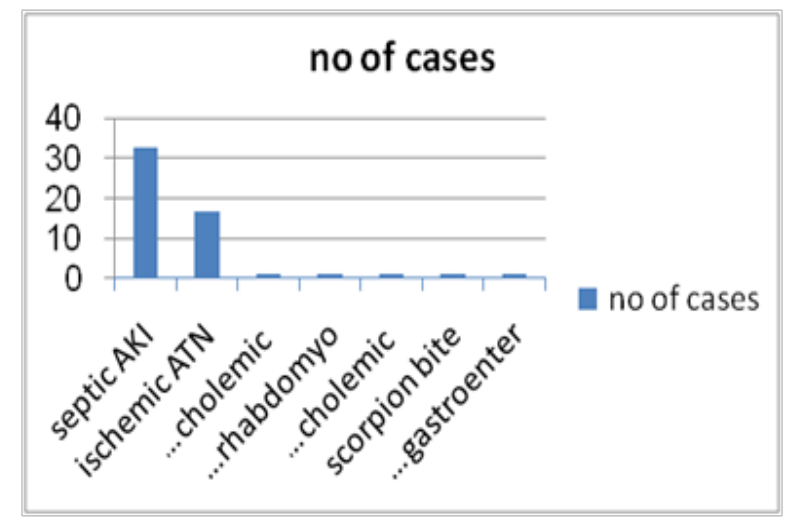

Figure 6 Classification of AKI general ICU patients.
Table 4 General ICU acquired AKI patients stratified by risk, injury, failure, loss and end stage renal disease (RIFLE) criteria

\begin{tabular}{lll}
\hline Stages & $\begin{array}{l}\text { No. and percent } \\
\text { of the patient }\end{array}$ & $\begin{array}{l}\text { Mortality all over } \\
\text { each group }\end{array}$ \\
\hline Risk & $20(36.4 \%)$ & $8 / 20(40 \%)$ \\
Injury & $27(49.1 \%)$ & $17 / 27(63 \%)$ \\
Failure & $8(14.5 \%)$ & $5 / 8(62.5 \%)$ \\
Total & 55 cases $(100 \%)$ & 30 cases
\end{tabular}

Table 5 ICU acquired AKI patients stratified by acute kidney network criteria

\begin{tabular}{lll}
\hline Stages & $\begin{array}{l}\text { No. and percent } \\
\text { of the patient }\end{array}$ & $\begin{array}{l}\text { Mortality all over } \\
\text { each group }\end{array}$ \\
\hline Stage I & $17(31 \%)$ & $7 / 17(41.2 \%)$ \\
Stage 2 & $30(54.5 \%)$ & $18 / 30(60 \%)$ \\
Stage 3 & $8(14.5 \%)$ & $5 / 8(62.5 \%)$ \\
Total & 55 cases (100\%) & 30 cases
\end{tabular}

Table 6 Comparison between mortality in different stages in RIFLE \& AKIN

\begin{tabular}{ll}
\hline $\begin{array}{l}\text { Stage of RIFLE Versus } \\
\text { Stage of AKIN }\end{array}$ & Mortality \\
\hline Risk vs. stage I & $40 \%$ v $41.2 \%$ \\
Injury vs. stage 2 & $63 \%$ v $60 \%$ \\
Failure vs. stage 3 & $62.5 \%$ v $62.5 \%$ \\
\hline
\end{tabular}

\section{Outcome}

Mortality rate allover general ICU patients: 140/500 (28\%). Mortality rate allover AKI patients in general ICU: $30 / 55$ (54\%). Fate of AKI general ICU patients on dialysis (Figure 7) 10 patients need dialysis 6 of them recovered \& 4 of them died. Septic cases $(128$ cases) classified into: no AKI (32\%), septic AKI (25.8\%), (Figure 8) acute on top of CKD (32\%), ESRD (10.2\%) (Table 7).

Table 7 Fate of sepsis according to renal impairment (128 cases)

\begin{tabular}{ll}
$\begin{array}{l}\text { Fate of sepsis according to } \\
\text { renal impairment }\end{array}$ & No of cases \\
No AKI & $41(32 \%)$ \\
Septic AKI & $33(25.8 \%)$ \\
Acute on top of CKD & $41(32 \%)$ \\
\hline ESRD & $13(10.2 \%)$ \\
& \\
total & 128 cases
\end{tabular}




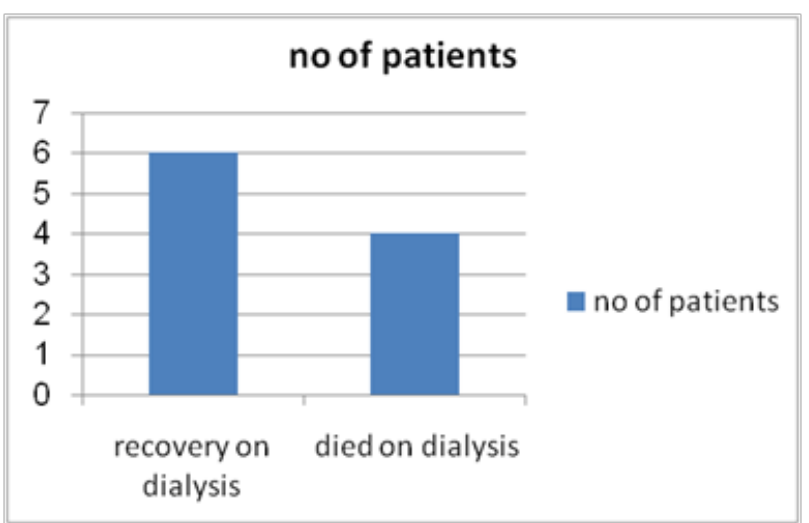

Figure 7 Fate of AKI general ICU patients on dialysis.

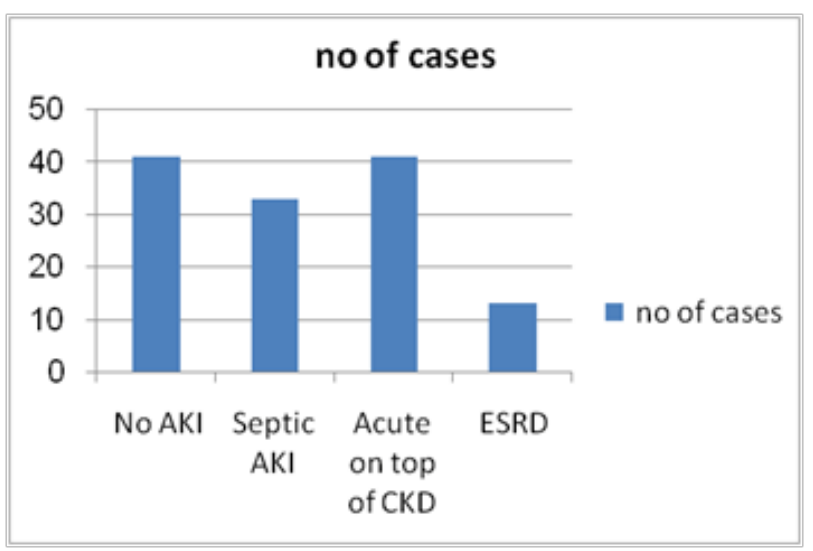

Figure 8 Fate of sepsis according to renal impairment.

\section{Discussion}

We conducted a prospective study to analyze the clinical Characteristics of the ICU acquired AKI in light of the AKIN and RIFLE classifications, and to evaluate the capacity of this system in predicting in hospital mortality of patients with AKI. In our study included 500 General ICU patients (classified according to renal impairment): 303 no AKI (60.6\%), 74 CKD (14.8\%), 55 AKI (11\%), 38 acute on top of CKD (7.6\%), 28 ESRD (5.6\%), 2 obstructive uropathy $(0.4 \%) \&$ Classified according to cause of admission: RTA 36 cases, FFH 30 cases, Toxo 60 cases, Botulism 10 cases, Snake bite 4 cases, Scorpion bite 1 case, Ischemic stroke 31 cases, Hemorrhagic stroke 25 cases, Meningitis 5 cases, Sepsis 128 cases (classified according to renal impairment into: no AKI (32\%), septic AKI (25.8\%), acute on top of CKD (32\%), ESRD (10.2\%), Hepatic 30 cases, DKA 26 cases, Obstetric 7 cases, Malignancy 21 cases, COPD 18 cases, IBD 1 case, GB 6 cases, TTP 2 cases, Cardiogenic 37 cases, Interstitial lung diseases 3 cases, Polymyositis 2 cases, Duchine muscle atrophy 1 case, Perforated peptic ulcer 1 case, Lupus pneumonitis 7 cases , Myasthenia gravis 2 cases, Auto immune hemolytic anemia 1 case, Blood diathesis 2 cases, CP 2 cases, Mucopoly saccharidosis 1 case.

Toxicological cases ( 75 cases) classified in to: Botulism 10 cases, Scorpion bite 1 case (developed AKI), Snake bite 4 cases, Organo phosphorus 27 cases (2 developed AKI), Tricyclic antidepressant (TCA) 4 cases, Tegretol 2 cases, Formalin 1 case, Depakin 1 case, Kerosin 2 cases, Zn phosphate 3 cases, Calmipam 4 cases, Insulin
2 cases, Lanoxin 6 cases, Tamol 1 case, Theophylline 1 case, Methanol 6 cases. Distribution of AKI in general ICU according to sex: Male patients 29/55 (52.7\%) Female patients 26/55 (47.3\%) \& age distribution was (18-85y). 10 patients need dialysis 6 of them recovered \& 4 of them died.

The prevalence of ICU-acquired acute renal failure was found to be $11 \%$ while Santos et al. ${ }^{8}$ reported that the incidence rateto be $22.6 \%$. Also, Clermont et al. ${ }^{9} \&$ Chawla et al. ${ }^{10}$ found theincidence of ARF in ICU patients to be $17 \%$ \& $18 \%$ respectively. In our study,septic AKIrepresented the most commonetiological diagnosis (60\%) then ischemic ATN (17/55) 31\%, cholemic nephrosis (1/55)1.8\%, rhabdomyolysis (1/55) $1.8 \%$, cardio renal syndrome (1/55) $1.8 \%$, scorpion bite (1/55) $1.8 \%$, gastroenteritis (1/55) $1.8 \%$ while Santos et al. ${ }^{8}$ reported that ischemic ATN was the most common cause of ARF in ICU with an incidence rate of $(48 \%)$. The Mortality rate allover general ICU patients: 140/500 (28\%) \& overall mortality rate allover AKI patients in general ICU: $30 / 55$ (54\%) similar to that $(51.9 \%)$ found by. ${ }^{11}$ Also Hoste et al. ${ }^{12}$ found a mortality rate of $56.7 \%$. Ricci and Ronco ${ }^{13}$ suggested that a 50-60\% crude mortality associated with ARF represents an acceptable level of performance to the health care system because as therapeutic capacity improves, the health care system will progressively admit and treat sicker and sicker patients with ARF.

In Our study 55 patients were met RIFLE criteria, 36.4\% classified as Risk, $49.1 \%$ classified as Injury, $14.5 \%$ classified as failure, while Hoste and colleagues who evaluated RIFLE as an epidemiological and predictive tool in 5,383 critically ill patients. ${ }^{12}$ They found that AKI occurred in a staggering $67 \%$ of patients, with $12 \%$ achieving class of R, $27 \%$ class I, and $28 \%$ class F. ${ }^{14}$ There was an increase in hospital mortality with increasing RIFLE class with patients who were class $\mathrm{R}$ having mortality rate of $40 \%$, patients who were class I having mortality rate of $63 \%$, patients who were class $F$ having mortality rate of $62.5 \%$,this progressive increase across different classes of RIFLE staging system agrees with Ricci et al. ${ }^{13}$ who analyzed data for more than 71,000 patients from published reports from August 2004 to June 2007 that have utilized RIFLE criteria and found that with Mortality was $18.9 \%, 36.1 \%$, and $46.5 \%$ in RIFLE class R, class I, and class F groups, respectively. ${ }^{15}$

In Our study 55 patients were met AKIN criteria , 31\% classified as stage $1,54.5 \%$ classified as stage $2,14.5 \%$ classified as stage 3 , in our study increasing AKIN stages correlated with increasing mortality, $41.5 \%, 60 \%$, and $62.5 \%$ in AKIN stage 1 , stage 2, and stage 3 groups, respectively, this agrees with Lopes et al. ${ }^{16}$ who found that mortality rate was in stage $1(34.6 \%)$, in stage $2(45 \%)$, and in stage $3(64.1 \%)$. When comparing corresponding degrees of AKI according to AKIN and RIFLE (stage 1versus 'risk'; stage 2versus 'injury'; stage 3 versus 'failure') no difference in mortality. Similar results were found by Bagshaw et al. ${ }^{17}$ and Ando et al. ${ }^{18}$

\section{Conclusion}

AKI is worsening problem, but its true incidence is in need of huge work, our work was 1st up to our knowledge in Alexandria to check its incidence hence planning for better patients' outcome. The recognition of AKI, using serum creatinine, often occurs hours to days after the initial insult hence the need for a recent specific marker that can detect a significant renal injury immediately. RIFLE criteria represent a simple tool for the detection and classification of AKI and for correlation with clinical outcomes. The AKIN criteria do not 
materially improve the sensitivity, robustness and predictive ability of the definition and classification of AKI.

\section{Acknowledgments}

None.

\section{Conflicts of interest}

The author declares there is no conflict of interest.

\section{Reference}

1. Uchino S, Kellum J, Bellomj R, et al. Acute Renal Failure in critically ill Patients, A multinational, multi-center study. JAMA. 2005;294(7):813818.

2. Bagshaw SM, Laupland KB, Doig CJ, et al. Prognosis for long-term survival and renal recovery in critically ill patients with severe acute renal failure: a population-based study. Crit Care. 9(6):R700-R709.

3. Kellum JA, Levin N, Bouman C, et al. Developing a consensus classification system for acute renal failure. Curr Opin Crit Care. 2002;8:509-514.

4. Bellomo R, Naka T, Baldwin I. Intravenous fluids and acid-base balance. Contrib Nephrol. 2004;144:105-118.

5. Bellomo R, Kellum JA, Ronco C. Defining and classifying acute renal failure: from advocacy to consensus and validation of the RiFLE criteria. Intensive Care Med. 2007;33(3):409-413.

6. Mehta RL, Kellum JA, Shah SV, et al. Acute kidney injury network: report of an initiative to improve outcomes in acute kidney injury. Crit Care. 2007;11(12):R31.

7. Seerapani Gopaluni, Simon Lines, Andrew JP Lewington. Acute kidney injury in the critically ill patient. Curren Anaesthesia \& Critical Care. 2010;21(2):60-64.
8. Santos WJ, Zanetta DM, Pires AC, et al. Patients with ischemic, mixed and nephrotoxic acute tubular necrosis in intensive care unit. crit care. 2006;10(2):68-76.

9. Clermont G, Acker C, Angus DC, et al. Renal failure in the ICU: comparison of the impact of acute renal failure and end stage renal disease on ICU outcomes. Kidney Int. 2002;62(3):986-996.

10. Chawla L, Abell L, Mazhari R, et al. Identifying critically ill patients at high risk for developing acute renal failure. Kid Int. 2005;68(5):2274 2280 .

11. Mehta RL, Pascual MT, Gruta CG, et al. Refining Predictive models in critically ill patients with acute renal failure. $J$ Am Soc Nephrol. 2002;13(5):1350-1357.

12. Hoste EA, Lameire NH, Vanholder RC, et al. Acute renal failure in patients with sepsis in a surgical ICU: predictive factors, incidence, comorbidity, and outcome. J Am Soc Nephrol. 2003;14(4):1022-1030.

13. Ricci Z, Ronco C. Kidney diseases beyond Nephrology--intensive care. Nephrol Dial Transplant. 200722(3):708-711.

14. Hoste EA, Clermont G, Kersten A, et al. RIFLE criteria for acute kidney injury are associated with hospital mortality in critically ill patients: A cohort analysis. Crit Care. 2006;10(3):R73.

15. Ricci Z, Cruz D, Ronco C. The RIFLE criteria and mortality in acute kidney injury: a systematic review. Kidney Int. 2008;73(5):538-546.

16. Lopes JA, Jorge S, Resina C, et al. Acute Kidney injury in patients with sepsis: a contemporary analysis. Int J Infect Dis. 2009;13(2):176-181.

17. Bagshaw SM, George C, Bellomo R. A comparison of the RIFLE and AKIN criteria for acute kidney injury in critically ill patients. Nephrol Dial Transplant. 2008;23(5):1569-1574.

18. Ando M, Mori J, Ohashi K, et al. A comparative assessment of the RIFLE, AKIN and conventional criteria for acute kidney injury after hematopoietic SCT. Bone Marrow Transplant. 2010;45(9):1427-1434. 